\title{
Developments and Perspectives in the Field of Sers Based Biosensors
}

\section{Lucian Baia*}

Department of the Condensed Matter Physics and Advanced Technologies \& Interdisciplinary Research Institute on Bio-Nano-Sciences, Babes-Bolyai University, M. Kogalniceanu 1, 400084 Cluj-Napoca, Romania

\section{Editorial}

I am delighted to take over as a new editor of the Journal of Biosensors and Bioelectronics (JBSBE). In the last years, one has observed a pronounced change of the way in which the scientific journals have published and disseminated the research articles. Thus, nowadays the printed versions of most journals become gradually a thing of the past, while the on-line versions and the unlimited access to journals are two aspects most often encountered on the research market. Nevertheless, the aim of each scientist is to publish papers in journals where their work is likely to have the highest impact. The reputation for quality of research and trustworthiness in the field of "Biosensors and Bioelectronics" of the Editorial Board Members of the JBSBE alongside with the used open access and peer-reviewed systems made up an excellent framework for the development of a real competition of valuable ideas that can permanently create opportunities for scientific debates and improvements. Additionally, since the papers from JBSBE are published by OMICS Publishing Group the authors will benefit by all of the offered special features (http://www.omicsonline.org/specialfeatures.php). With all these aspects in mind, one can infer that the quality of the journal will be closely related to the conducted research in terms of both scientific accuracy as well as topic of interest. In the case of biosensors field, one of the most important task is related to the biochemical and biomedical sensing applications.

One of the main challenges faced by biodetection systems is the ability to detect and identify a large range of target analytes at low concentrations and in short times. Surface-Enhanced Raman Scattering (SERS) remarks itself as a powerful analytical tool that was successfully used in various biochemical and biomedical sensing applications [13]. This technique originates from the Raman spectroscopy, which provides information about the structures under investigation at the molecular level, but additionally takes the benefit of an important enhancement of the Raman signal as a result of positioning the scatterer in the very close vicinity of a nanoscale-roughed metallic surface, particularly a noble metal surface. Two main mechanisms can simultaneously contribute to the overall SERS enhancement, a long-range electromagnetic effect $\left(\sim 10^{2}-10^{6}\right)$ and a short-range chemical effect $\left(\sim 10-10^{2}\right)$ [3-6]. The chemical mechanism contributes to enhancement through chemisorption of the molecule to the noble metal surface and can vary between substrates, substrate adsorption sites, and adsorbed molecules, while the electromagnetic mechanism is a wavelength-dependent effect that arises from the excitation of the Localized Surface Plasmon Resonance (LSPR), i.e. collective oscillation of conduction electrons, which occur in noble metal nanoparticles (NPs), sharp metal tips, or roughened metal surfaces, and enhances the incident electric field intensity [3,7]. Concerning the SERS detection via LSPR excitation it is extremely important to note that, in this way, a strong SERS signal can be obtained from any SERS-active molecule located in the area where the electromagnetic field is enhanced. This means that a single source can be used for multiple labels. Besides the amplification of the signal obtained by the two described mechanisms another additional enhancement (up to $10^{6}$ ) can be acquired when the laser excitation frequency is close to both the electronic absorption of the analyte and the Plasmon Resonances of the noble metal surface, the overall gain of the Raman signal reaching $10^{14}$ [3].
From the long range of SERS applications with a biosensing character one remarks the capacity of detecting ultrasensitive limits, including single molecule sensitivity, pathogens that include bacteria and viruses as well as the ability to discriminate between types and strains of pathogens, including pathogens with gene deletions $[1,2,5]$. Also, in the last few years a series of studies have been reported, in which SERS was employed in biosensing applications on cellular and in vivo systems, e.g. in vivo cancer marker detection and in vivo glucose concentration sensing $[2,3]$.

Basically, there are two SERS configurations that have been used in biosensing $[3,4]$. The first one is the intrinsic format, in which the analyte is directly located on the nanostructured surfaces from where the enhanced signal of the biomolecule is directly acquired, e.g. small molecule, DNA strand, or protein. To enable a well established specificity of detection an indirect detection configuration can also be employed. In this case the molecule under investigation is immobilized onto a nanostructured surface and the Raman spectral differences before and after capture of the specimen are recorded, e.g. antibodies, aptamers, or related molecules. The second configuration is the extrinsic format, in which a Raman reporter molecule is used to generate a signal for detection $[3,4]$. The analyte in this situation is associated with a molecule that exhibits an intense and distinguishable Raman signature, e.g. commercially available fluorescent dye, and the recorded SERS spectrum is further used for sensing. One should emphasize that each of the above described configurations can offer special benefits in sensing capacities $[3,4]$.

In addition to the most often explored parameters in biosensing field (such as rapid and sensitive detection, reduction or elimination of sample preparation, obtaining reproducible results, cost effectiveness, simplicity of use under most conditions) the techniques used in the last decades tried to overcome other limitations related to the detection and discrimination of specimens in complex biological media. Thanks to the narrower spectral widths SERS represents the appropriate method able to minimize the overlap between different labels and to increase the multiplex capability. Moreover, SERS technique is very often applied in detecting and identifying biological species and their changes in dynamic systems like microfluidics media [8].

A new emerging branch of SERS is the Tip-Enhanced Raman Spectroscopy (TERS), in which the tip is either a metal coated tip, as used in Scanning Probe Mycroscopy (SPM), or a silver/gold wire. The

*Corresponding author: Lucian Baia, Department of the Condensed Matte Physics and Advanced Technologies \& Interdisciplinary Research Institute on Bio-Nano-Sciences, Babes-Bolyai University, M. Kogalniceanu 1, 400084, ClujNapoca, Romania, E-mail: lucian.baia@phys.ubbcluj.ro

Received March 30, 2012; Accepted April 01, 2012; Published April 05, 2012

Citation: Baia L (2012) Developments and Perspectives in the Field of Sers Based Biosensors. J Biosens Bioelectron 3:e110. doi:10.4172/2155- 6210.1000e110

Copyright: () 2012 Baia L. This is an open-access article distributed under the terms of the Creative Commons Attribution License, which permits unrestricted use, distribution, and reproduction in any medium, provided the original author and source are credited. 
Citation: Baia L (2012) Developments and Perspectives in the Field of Sers Based Biosensors. J Biosens Bioelectron 3:e110. doi:10.4172/2155$6210.1000 \mathrm{e} 110$

Page 2 of 2

apex of a laser-irradiated metal tip captures incident light and generates a strong LSPR for SERS. The simultaneous use of both SERS chemical fingerprinting and SPM imaging ensures remarkable advantages, i.e. the high resolution SERS images, high sensitivity and contrast, lateral resolution down to a few tens of nanometers and the fact that the analyte does not need to be in direct contact with the SERS-active substrate, that defines TERS as one of the most sensitive and "full of information" technique for the future of biochemistry and biomedicine fields $[2,3]$.

The steps made in the development of the SERS based biosensor were huge, but imminently a question arises: what comes next? The perspectives can follow several directions. The first one is closely related to the role of the SERS substrate that strongly depends on its inherent properties like size, shape, or interparticle spacing. Therefore, the fabrication of novel noble metal nanoarchitectures capable to tune the Plasmon Resonances and to further deliver increasingly higher enhancements of the Raman signal as well as reproducibility and stability to the substrate, which still represent drawbacks for certain substrates, will certainly be one of the future tasks. An important progress can also be closely related with the increasing of the enhanced Raman signal by acting with external factors on the noble metal surface with the desired morphology, e.g. temperature, light, magnetic field, etc. In the meantime one expects the improvement of TERS or even the development of other new techniques that would allow biomolecules analysis by SERS, without being in contact with the substrate. SERS based biosensors will be deeply employed in the future in clinical, cellular and in vivo applications. Nevertheless, in order to obtain accurate results in biosensing both in laboratory as well as under field conditions it is imperious necessary to develop in the forthcoming period biosensing platform technologies capable to substantially improve the biomedical domain, in particular, and our social level, in general.

\section{References}

1. Kho KW, Fu CY, Dinish US, Olivo M (2011) Clinical SERS: are we there yet? J Biophotonics 4: 667-684.

2. Nafie LA (2011) Recent advances in linear and nonlinear Raman spectroscopy Part V. J. Raman Spectrosc 42: 2049-2068.

3. Bantz KC, Meyer AF, Wittenberg NJ, Im H, Kurtuluş O, et al. (2011) Recent progress in SERS biosensing. Phys Chem Chem Phys 13: 11551-11567.

4. Xie W, Qiu P, Mao C (2011) Bio-imaging, detection and analysis by using nanostructures as SERS substrates. J Mater Chem 21: 5190-5202.

5. Tripp RA, Dluhy RA, Zhao Y (2008) Novel nanostructures for SERS biosensing Nano Today 3: 31-37.

6. Nafie LA (2010) Recent advances in linear and nonlinear Raman spectroscopy Part IV. J Raman Spectrosc 41: 1566-1586.

7. Tokonami S, Yamamoto Y, Shiigi H, Nagaoka T (2012) Synthesis and bioanalytical applications of specific-shaped metallic nanostructures: a review. Anal Chim Acta 716: 76-91.

8. Huh Y, Chung A, Erickson D (2009) Surface enhanced Raman spectroscopy and its application to molecular and cellular analysis. Microfluid Nanofluid 6: 285-297. 